\title{
Understanding the past to appropriately guide us in the future
}

\author{
Charles Beale, $M D,{ }^{a}$ and Peter Tilkemeier, $M D, M M M^{b}$ \\ a Brown University, Providence, RI \\ ${ }^{\text {b }}$ Greenville Health System, Greenville, SC
}

Received Jul 23, 2015; accepted Jul 24, 2015

doi: 10.1007/s12350-015-0257-6

\section{See related article, pp. 680-689}

In recent years up until the early 2000s, the utilization of cardiovascular imaging has increased, particularly Myocardial Perfusion Imaging (MPI), subsequently MPI has come under increased scrutiny due to concern of overuse and therefore increasing the cost of healthcare delivery. Clinicians previously viewed the value of diagnostic tests by solely evaluating the benefits of testing but neglected to evaluate the potential detrimental effect of performing a diagnostic test in a low risk population. This has led to the publication of the Appropriate Use Criteria (AUC) by the American College of Cardiology Foundation along with cosponsoring organizations in 2005, and revised in 2009 . $^{1}$ The AUC is designed to mitigate unnecessary testing, subsequently reducing cost, focusing on higher risk populations, and ultimately improving patient care. The AUC implementation has presented new challenges to the medical community as physician understanding or knowledge of the AUC can be variable. Compliance with the AUC in clinical practice is currently transforming care and will continue to do so in the future in yet unknown ways.

In this Issue of the Journal of Nuclear Cardiology, Dr. Elgendy and colleagues preformed a meta-analysis to review and subsequently validate the impact of the AUC by reviewing 22 published studies accounting for 23,443 tests. They concluded patients who had inappropriate testing were more unlikely to demonstrate

Reprint requests: Peter Tilkemeier, MD, MMM, Greenville Health System, Greenville, SC; ptilkemeier@gmail.com

J Nucl Cardiol 2016;23:693-4.

1071-3581/\$34.00

Copyright (C) 2015 American Society of Nuclear Cardiology. ischemia. There was substantially heterogeneity of the studies used, including differences in community-based practice vs academic centers, single centers vs multicenter and prospective vs retrospective, for example. This diversity is important as the findings become meaningful in the real world settings in which we practice today. Inappropriate testing in the reviewed studies ranged from 5\% to $46 \%$. This large range of variability is also suggestive of the challenges faced in determining which tests are unnecessary, findings supported by the study of Ye et al. They reviewed the consistency of interns, internal medicine hospitalists, or cardiology fellows in their ability to decipher inappropriate testing. They concluded cardiology fellows were just as likely to agree with interns as they would other physicians at the same level of training in comparison to cardiology attendings. ${ }^{2}$ The meta-analysis by Elgendy extends this concept to further state inappropriate testing occurs at the same frequency between the cardiology and non-cardiology physicians. Dr. Elgendy and colleagues do remark education alone is not effective, nor has the rate of clinically irrelevant MPI's decreased over time. There are many plausible explanations of these findings, including ambiguity in the AUC guidelines

\footnotetext{
${ }^{1}$ Hendel RC, Berman DS, Di Carli MF, et al. ACCF/ASNC/ ACR/AHA/ASE/SCCT/ SCMR/SNM 2009 Appropriate Use Criteria for Cardiac Radionuclide Imaging: a report of the American College of Cardiology Foundation Appropriate Use Criteria Task Force, the American Society of Nuclear Cardiology, the American College of Radiology, the American Heart Association, the American Society of Echocardiography, the Society of Cardiovascular Computed Tomography, the Society for Cardiovascular Magnetic Resonance, and the Society of Nuclear Medicine. J Am Coll Cardiol. 2009;53:2201-2229.

2 Ye S, Rabbani, L, Kelly, C, et al. Can Physicians Identify Inappropriate Nuclear Stress Test?: An Examination of InterRater Reliability for the 2009 Appropriate Use Criteria for Radionuclide Imaging. Circulation Cardiovascular Quality Outcomes., Jan. 6, 2015:8 23-29
} 
leading to their misunderstanding, lack of knowledge of the guidelines or inadequate tools to support their implementation in clinical practice as examples.

Regardless of the challenges in determining appropriateness, Elgendy et al concluded $14.8 \%$ of MPI testing were inappropriate. ${ }^{3}$ This number equates to approximately 1 in 6.5 tests being inappropriate. The implication further leads to the inefficiency of cost per study. The cost effectiveness of SPECT myocardial perfusion imaging has been reviewed by Hayes-Brown et al. As per their assessment, the annualized cost per one major adverse cardiac event (MACE) predicted differed $\$ 1,267,313$ based on a difference in appropriate patients at the cost per MACE of $\$ 268,682$ vs. $\$ 1,535,995$ with inappropriate use. ${ }^{4}$ Their review, however, was limited by a relatively small sample size of 1511 patients, with a disproportionate amount of inappropriate testing of $45 \%$. In anticipation of the high cost of medical services of the emerging Baby Boomer Population, and to support the value of healthcare delivery, payers will attempt to "cut the fat" of excessive spending and will make payments of such studies more challenging unless we can alter the current course.

Furthermore Elgendgy subsequently reported inappropriate testing has a low yield for discovering ischemia, and therefore validating the AUC. Of note the AUC is not intended to supersede clinical judgment; however, such a high incidence of inappropriate MPI testing which does not demonstrate ischemia suggests that clinical judgement may not be superior. The use of prior authorization was also evaluated and it appears to have no effect on the number of unnecessary tests. Instead of creating more barriers to clinicians, system- based support systems may be helpful to alleviate confusion and clinician uncertainty.

The challenges to clinicians will not end in only prior authorizations or withholding of payments. The DoctorPatient relationship has changed dramatically from a previously predominately paternalistic one, to one centered rightfully on patient autonomy. This has contributed to the addition of patient satisfaction as a marker of reimbursement and the Centers for Medicare and Medicaid Service in 2016 will withhold up to $2 \%$ of Medicare reimbursements depending on this performance indicator. These will present yet another challenge, as clinician may need to better manage patient expectations based on previous norms of stress testing.

Nonetheless, a reasonable conclusion is the use of AUC criteria will increase value to the health care system by reducing the number of rarely appropriate tests performed without sacrificing quality of care. There are, however, limitations of the AUC which have yet to be delineated. Integration of the AUC in decision support tools may help increase the current rates of appropriate testing as implementation of the AUC in clinical practice is underutilized or misunderstood. Future studies will be needed to understand the barriers faced in educating clinicians ordering testing, as opposed to creating barriers such a more challenging reimbursements, or even litigation. We need to embrace the technologies available to us moving forward to implement guideline informed clinical decision support tools at the point of care as a tool to break the cycle. Well informed, comprehensive, individualized clinical guidance will seamlessly integrate the appropriate choice into optimized daily patient care.

\footnotetext{
3 Elgendy, I, Mahmoud, A, et al. "Outcomes after inappropriate nuclear myocardial perfusion imaging: A meta-analysis. Journal Of Nuclear Cardiology, Date Submitted May 15, 2015 ${ }^{4}$ Hayes-Brown, Kathleen, et al. The Impact of appropriate use on the cost effectiveness of SPECT Myocardial Perfusion Imaging in the Community Setting. Journal of American Cardiology, Volume 63, Issue 12.
} 